\title{
Metodología de gestión basada en lean construction y pmbok; Para mejorar la productividad en proyectos de construcción.
}

Management methodology based on lean construction and pmbok; To improve productivity in construction projects.

\author{
Margot Millones Mateus \\ Universidad Nacional de San Agustín. Arequipa Perú.
}

\section{INFORMACIÓN}

\section{Historia del Artículo}

Recepción: 10/03/2020

Revisión: 05/05/2020

Aceptación: 20/05/2020

Palabras Clave
Lean Construction, PMI,
Productividad

\section{Key Words}

Lean Construction, PMI, Productivity

\section{DOI}

https://doi.org/10.35286/veritas. v21i2.276

\begin{abstract}
RESUMEN
El artículo expone resultados, acerca de procesos vinculados con la gestión de proyectos, con el objetivo de proponer una metodología basada en Lean Construction y en los fundamentos de la Guía del PMBOK; que permita mejorar la productividad en proyectos de construcción. La metodología obtenida de integrar el escenario de la Planificación Last Planner, al grupo de procesos de planificación, así como al grupo de procesos de monitoreo y control del escenario del PMI (Fundamentos de la Guía del PMBOK); logrará optimizar los factores que afectan la planificación, el monitoreo y control de proyectos de construcción mejorando su productividad; reflejándose en la mejora de la calidad, tiempo y costo del proyecto. La optimización del proceso de Perfilado de la subrasante sin aporte de material generó grandes resultados (outputs) como la reducción del tiempo (plazo de ejecución) de 98 días a 68 días y la reducción del costo de $\mathrm{S} /$. $255,851.59$ a S/. 230,061.70; estos outputs son por unidad de consumo (inputs) que corresponde a los recursos administrados eficientemente (Mano de obra, equipo y materiales) y a un eficaz control de calidad.
\end{abstract}

\begin{abstract}
The article presents the results about processes related to project management, with the aim of proposing a methodology based on Lean Construction and the fundamentals of the PMBOK Guide; that allows to improve the productivity of construction projects. The methodology obtained from integrating the Last Planner Planning scenario, the planning process group, as well as the PMI scenario monitoring and control process group (Fundamentals of the PMBOK Guide); will optimize the factors that affect the planning and monitoring and control of construction projects by improving their productivity; reflected in the improvement of the quality, time and cost of the project. The optimization of the Sub-profile profiling process without material input generated great results (outputs) such as the reduction of the time (execution period) from 98 days to 68 days and the reduction of the cost of S/. 255,851.59 to S/. 230,061.70; these outputs are per unit of consumption (inputs) corresponding to the efficiently managed resources (labor, equipment and materials) and an effective quality control.
\end{abstract}

\section{INTRODUCCIÓN}

El sector construcción en el Perú, es uno de los ejes principales de su desarrollo; por ser uno de los más dinámicos que impulsan la economía.

La productividad es la capacidad de una organización para agregar valor a los recursos que consume, consiste en hacer más productos o servicios con menos recursos que implica la utilización más eficiente de los mismos. Para mejorarla es necesario medirla, posterior a ello analizar cuáles son los factores que la afectan; para finalmente seleccionar los que tienen mayor incidencia y mejorarla.

Las pérdidas que se generan durante la ejecución de actividades de conservación vial de carreteras, se deben a diferentes causas, una de las más importantes radica en la elaboración no optimizada de proyectos que genera pérdidas; las que son muy frecuentes y tienen un impacto económico considerable. La aplicación del Lean Construction, permite realizar un análisis de tiempos productivos (TP), contributorios (TC) y no contributorios (TNC); con la finalidad de determinar la eficiencia de los procesos y eliminar las pérdidas que no agregan valor.

Con la finalidad de incrementar la transparencia, reducir la incertidumbre y la variabilidad existente debido a la complejidad propia de los proyectos de construcción; estos deberían ser gestionados como un sistema de producción. En este sentido el objetivo es mejorar la productividad en proyectos de construcción mediante la aplicación de una metodología de gestión de proyectos basada en el Lean Construction (planificación Last Planner) y en los fundamentos de la guía PMBOK del Project Management Institute. 


\section{OBJETIVO}

Proponer una metodología de gestión basada en Lean Construction y PMBOK; para mejorar la productividad en proyectos de construcción.

\section{METODOLOGÍA}

\section{Diseño}

El tipo de diseño es no experimental, porque no se manipulan variables, solo se observan los hechos en la realidad y es de carácter transeccional; porque los hechos se observan en un determinado momento.

\section{Modelo propuesto}

Es el resultado de integrar el escenario de la planificación Last Planner al Grupo de procesos de planificación y al Grupo de procesos de monitoreo y control del escenario del PMI (Fundamentos de la Guía PMBOK).

Para ello, los tres primeros niveles de la planificación Last Planner son integrados al grupo de procesos de planificación del PMI (Fundamentos de la Guía PMBOK), por otro lado, el cuarto nivel del Last Planner se integra al grupo de procesos de monitoreo y control del PMI. De esta manera la planificación Last Planner, permitirá mejorar la productividad de los proyectos de construcción, que serán traducidos en flujos de trabajo continuos; con mejoras en la calidad, tiempo y costo del proyecto. En la Figura 1, se puede apreciar la representación gráfica de la metodología propuesta.

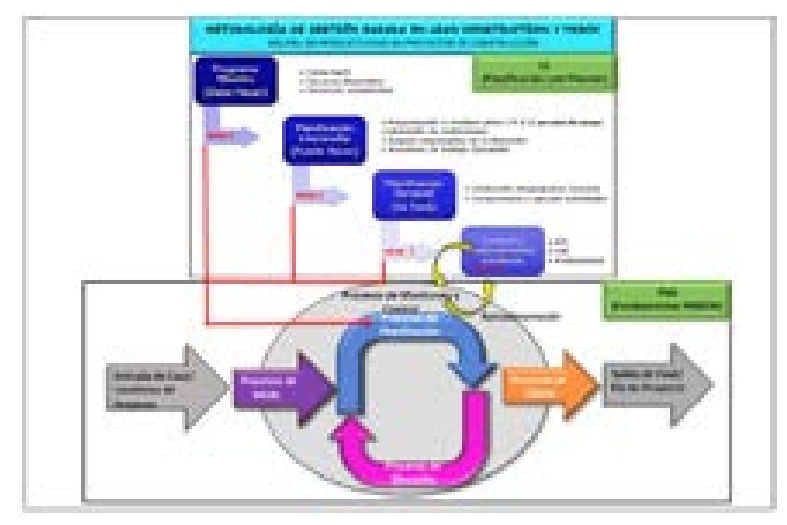

Fig. 1: Representación gráfica de la metodología propuesta Lean Construction y PMBOK. Fuente: Adaptado de (Guía del PMBOK, 2017) y (Tapia y González, 2014)

\section{ANÁLISIS}

\section{a. Recolección de datos} datos:

Se ha utilizado las siguientes técnicas de recolección de

\section{Técnicas de análisis documental}

Los documentos analizados se encuentran en la bibliografía

\section{Observación directa}

Se obtuvo información sobre los criterios de verificación de los factores que influyen en la productividad de los proyectos analizados; información que ha sido plasmada en las listas de chequeo - checklist.

\section{Cartas de proceso}

Representadas por símbolos interconectados, que representan la secuencia de los eventos individuales de la actividad en estudio y que proporciona una ayuda visual del proceso en su conjunto.

\section{b. Análisis de datos}

Primero se obtuvieron datos aislados, que son el resultado de la aplicación de las técnicas seleccionadas para la recolección de datos.

Para el análisis de la productividad en proyectos de construcción, se consideraron tres proyectos de conservación vial ejecutados en el año 2015 en el departamento de Arequipa.

Los factores que afectaron la productividad, están relacionados con la planificación, monitoreo, control y productividad. En cuanto a la planificación, no se consideran los riesgos potenciales, el tiempo, costo ni alcance del proyecto; que dan origen al incumplimiento de los plazos de ejecución y a la generación de sobre costos.

En el monitoreo y control a operación, no se cuenta con un plan de mantenimiento de equipo mecánico y no se realiza el control de los rendimientos de las actividades; lo que genera poca eficiencia en el uso de equipos.

Finalmente, en lo relacionado a la productividad, no son compatibles los rendimientos de las actividades de conservación vial y no se considera la mejora de procesos.

De los 3 proyectos de conservación vial analizados, se eligió el que presenta mayor influencia de los factores en su productividad, para la validación del Modelo propuesto.

\section{c. Presentación de los datos}

Seguidamente, se específica la validación de acuerdo a la estructura de la metodología de gestión propuesta, en los Grupos de procesos donde se integró la planificación Last Planner.

\section{Grupo de procesos de planificación}

\section{Gestión del cronograma del proyecto}

- Nivel 1.- Programa maestro - Cronograma del proyecto

Lo que se "Debe hacer" (Cronograma del proyecto versión original), corresponde al programa maestro. Tabla 1. 
Tabla 1: Resumen del Programa maestro - versión original. Fuente: Elaboración propia

\begin{tabular}{lccccc}
\hline \multicolumn{5}{c}{ Programa maestro - versión original } \\
\hline Entregables & $\begin{array}{c}\text { Plazo de } \\
\text { ejecución }\end{array}$ & $\begin{array}{c}\text { Fecha de } \\
\text { inicio }\end{array}$ & $\begin{array}{c}\text { Fecha } \\
\text { termino }\end{array}$ & $\begin{array}{c}\text { Fuentes } \\
\text { de trabajo }\end{array}$ & $\begin{array}{c}\text { Presupuesto } \\
\mathrm{S} / .\end{array}$ \\
\hline $\begin{array}{c}\text { Gestión del } \\
\text { proyecto }\end{array}$ & $98 \mathrm{dc}$ & $25 / 08 / 2016$ & $05 / 12 / 2016$ & 1 & 255.851 .59 \\
$\begin{array}{c}\text { Ejecución del } \\
\text { proyecto }\end{array}$ & & & & & \\
\hline
\end{tabular}

Dentro de las actividades de conservación vial, la actividad de Perfilado de la Subrasante sin aporte de material; es considerada una actividad crítica que determina el plazo de ejecución del proyecto.

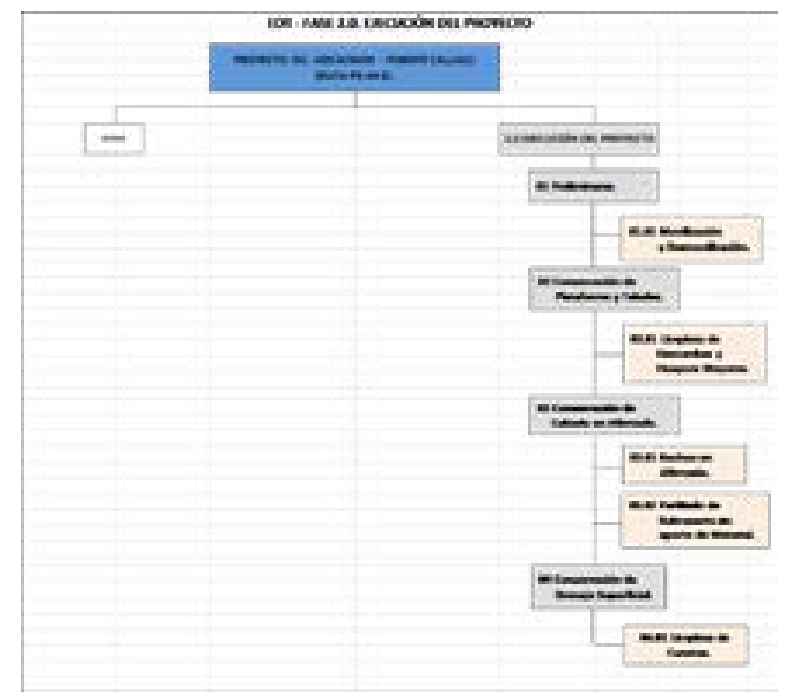

Fig. 2: Actividades de Conservación Vial (Mantenimiento Rutinario). Fuente: Elaboración propia

En la actividad de Perfilado de la Subrasante sin aporte de material, el equipo mecánico tiene una intervención del $90 \%$; logrando un rendimiento diario de $1.6 \mathrm{~km} /$ día.

El Subproceso crítico es el Riego de Subrasante (la cantidad disponible de agua es de $72 \mathrm{~m} 3 /$ día), con 9 viajes por día de la cisterna de 2000 glns; que implica $45.5 \mathrm{~min}$. $\mathrm{Si}$ se requiere $144 \mathrm{~m} 3 /$ día de agua, el subproceso debe optimizarse.

En la Figura 3, se puede apreciar el análisis del Proceso de Perfilado de la subrasante sin aporte de material y del subproceso de riego. (Sin aplicación de la Metodología).

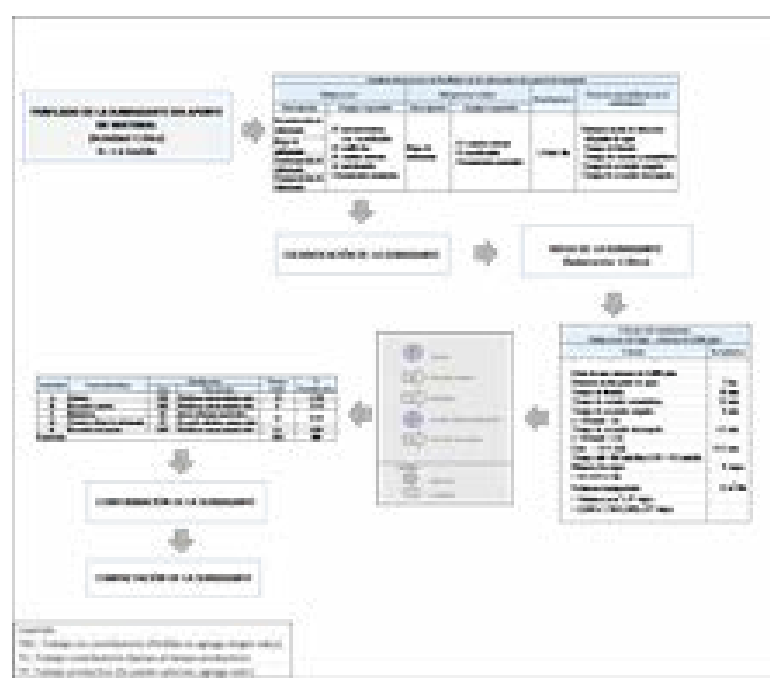

Fig. 3: Análisis del Proceso de Perfilado de Subrasante sin aporte de material (Sin aplicación de la Metodología)

El agua disponible es de $72 \mathrm{~m} 3$ /día, menor al requerido (144 m3/día). La optimización se realizó incrementando de 01 a 02 cisternas (incluye motobomba). El rendimiento diario del proceso se incrementó de $1.6 \mathrm{~km} /$ día a $3.2 \mathrm{~km} /$ día.; según se detalla en la Tabla 2.

Tabla 2: Comparación entre la versión original y el sinceramiento de metrados (incluye optimización de proceso). Fuente: Elaboración propia

\begin{tabular}{|c|c|c|c|c|c|c|c|}
\hline \multirow{2}{*}{ Entregables } & \multirow{2}{*}{$\begin{array}{l}\text { Plazo de } \\
\text { ejecucion }\end{array}$} & \multirow{2}{*}{$\begin{array}{l}\text { Fecha de } \\
\text { inicio }\end{array}$} & \multirow{2}{*}{$\begin{array}{l}\text { Fecha de } \\
\text { termino }\end{array}$} & \multirow{2}{*}{$\begin{array}{l}\text { Fuentes de } \\
\text { trabajo }\end{array}$} & \multirow{2}{*}{ Presupuesto } & \multicolumn{2}{|c|}{$\begin{array}{l}\text { Proceso Perfilado de la } \\
\text { subrasante }\end{array}$} \\
\hline & & & & & & Rendimiento & $\begin{array}{c}\text { Costo por } \\
\mathrm{km} .\end{array}$ \\
\hline \multicolumn{8}{|c|}{ Programa Maestro - Versión Original } \\
\hline $\begin{array}{l}\text { Gestión del } \\
\text { proyecto }\end{array}$ & \multirow[b]{2}{*}{$98 \mathrm{dc}$} & \multirow[b]{2}{*}{ 25/08/2016 } & \multirow[b]{2}{*}{ 05/12/2016 } & \multirow[b]{2}{*}{1} & \multirow[b]{2}{*}{$255,851.59$} & \multirow[b]{2}{*}{$1.6 \mathrm{~km} / \mathrm{día}$} & \multirow[b]{2}{*}{$2,124.28$} \\
\hline $\begin{array}{l}\text { Ejecucion del } \\
\text { proyecto }\end{array}$ & & & & & & & \\
\hline \multicolumn{8}{|c|}{ Programa Maestro - Sinceramiento de metrados (incluye optimización de proceso) } \\
\hline $\begin{array}{l}\text { Gestión del } \\
\text { proyecto }\end{array}$ & \multirow[b]{2}{*}{$68 \mathrm{dc}$} & \multirow[b]{2}{*}{ 25/08/2016 } & \multirow[b]{2}{*}{$31 / 10 / 2016$} & \multirow[b]{2}{*}{2} & \multirow[b]{2}{*}{$230,061.70$} & \multirow[b]{2}{*}{$3.2 \mathrm{~km} / \mathrm{día}$} & \multirow[b]{2}{*}{$1,697.34$} \\
\hline $\begin{array}{l}\text { Ejecucion del } \\
\text { proyecto }\end{array}$ & & & & & & & \\
\hline
\end{tabular}

- Nivel 2.- Planificación intermedia - Lookahead planning

Lo que se "Puede hacer", se determinó en base al programa maestro; de acuerdo a los siguientes criterios:

- Programación a mediano plazo (4 semanas) Se definió como intervalo de tiempo 4 semanas.

- Liberación de restricciones y asignación de responsables de la liberación

Para determinar lo que se "Hará", se identificaron las actividades que se ejecutarán en el intervalo establecido; luego se realizó el análisis de restricciones según se puede ver en la Tabla 3. 
Tabla 3: Análisis de restricciones. Fuente: Elaboración propia

\begin{tabular}{|c|c|c|c|c|c|c|c|c|c|c|}
\hline \multirow[b]{2}{*}{$\mathrm{N}^{\circ}$} & \multirow[b]{2}{*}{ Actividades } & \multicolumn{7}{|c|}{ Restricción } & \multicolumn{2}{|c|}{ Responsable } \\
\hline & & $\begin{array}{c}\text { Fecha de } \\
\text { inicio } \\
\text { Look ahead }\end{array}$ & Cant. & Unid. & Descripción & $\begin{array}{l}\text { Especificaciones } \\
\text { técnicas }\end{array}$ & $\begin{array}{l}\text { Lugar de } \\
\text { entrega }\end{array}$ & $\begin{array}{l}\text { Fecha } \\
\text { requerida en } \\
\text { campo }\end{array}$ & Área & Personal \\
\hline 1 & \multicolumn{10}{|c|}{ Gestión del proyecto } \\
\hline & $\begin{array}{l}\text { Adquisiciones de bienes y } \\
\text { servicios }\end{array}$ & $1 / 97 / 2016$ & & & $\begin{array}{l}\text { Bienes y servicios } \\
\text { requeridos }\end{array}$ & & $\begin{array}{l}\text { Campamente } \\
\text { Pulpera }\end{array}$ & $14 / 09 / 2016$ & Logística & $\mathrm{CF}$ \\
\hline 2 & \multicolumn{10}{|c|}{ Ejecución del proyecto } \\
\hline & $\begin{array}{l}\text { Movilización de equipo } \\
\text { mecánico }\end{array}$ & $15 / 09 / 2016$ & 6.00 & Und & $\begin{array}{l}\text { Equipo mecánico } \\
\text { requerido }\end{array}$ & Operativo & $\begin{array}{l}\text { Campamento } \\
\text { Pulpera }\end{array}$ & $14 / 09 / 2016$ & $\begin{array}{l}\text { Logística } \\
\text { EEM }\end{array}$ & $\begin{array}{l}\text { CF } \\
\text { JG }\end{array}$ \\
\hline & $\begin{array}{l}\text { Desmovilización de equipo } \\
\text { mecánico (CV/CF) }\end{array}$ & $16 / 10 / 2016$ & 2.00 & Und & $\begin{array}{l}\text { Disponibilidad de } \\
\text { cama baja }\end{array}$ & & $\begin{array}{l}\text { Campamento } \\
\text { Chiguata }\end{array}$ & $16 / 10 / 2016$ & $\begin{array}{l}\text { Logística } \\
\text { EEM }\end{array}$ & $\begin{array}{l}\text { CF } \\
\text { JG }\end{array}$ \\
\hline & $\begin{array}{c}\text { Desmovilización de equipo } \\
\text { mecánico (CV/TN/MN/ } \\
\text { RLL/CC) }\end{array}$ & $23 / 10 / 2016$ & 3.00 & Und & $\begin{array}{l}\text { Disponibilidad de } \\
\text { cama baja }\end{array}$ & & $\begin{array}{l}\text { Campamento } \\
\text { Chiguata }\end{array}$ & $23 / 10 / 2016$ & $\begin{array}{l}\text { Logística } \\
\text { EEM }\end{array}$ & $\begin{array}{l}\text { CF } \\
\text { JG }\end{array}$ \\
\hline
\end{tabular}

- Inventario de trabajo ejecutable (ITE)

Las actividades que se encuentran libres de restricciones y que fueron identificadas en la planificación intermedia, se consignaron en el ITE. El resumen de los mismos se muestra en la Tabla 4.

Tabla 4: Resumen de Inventario de trabajo ejecutable (ITE)

\begin{tabular}{|c|c|c|c|c|c|c|}
\hline \multirow{2}{*}{$\mathrm{N}^{\circ}$} & \multirow{2}{*}{ Actividades } & \multicolumn{2}{|c|}{ Fecha } & \multicolumn{2}{|c|}{ Semana } & \multirow[t]{2}{*}{ ¿Entran a la semana? } \\
\hline & & Inicio & Termino & Inicio & Fin & \\
\hline 1 & \multicolumn{6}{|c|}{ Gestión del proyecto } \\
\hline 1.1 & Iniciación & $25 / 08 / 2016$ & $26 / 08 / 2016$ & Semana 00 & Semana 00 & Sí \\
\hline 1.2 & Plan del proyecto & $27 / 08 / 2016$ & $04 / 09 / 2016$ & Semana 00 & Semana 01 & Sí \\
\hline 1.3 & Ejecución & 05/09/2016 & $31 / 10 / 2016$ & Semana 02 & Semana 10 & Sí \\
\hline 1.4 & Control & $12 / 09 / 2016$ & $31 / 10 / 2016$ & Semana 03 & Semana 10 & Sí \\
\hline 1.5 & Riesgos & $27 / 08 / 2016$ & $27 / 08 / 2016$ & Semana 00 & Semana 00 & Sí \\
\hline 1.6 & Cierre del proyecto & $25 / 10 / 2016$ & $31 / 10 / 2016$ & Semana 09 & Semana 10 & Sí \\
\hline 2 & \multicolumn{6}{|c|}{ Ejecución del proyecto } \\
\hline 1.00 & \multicolumn{6}{|c|}{ Preliminares } \\
\hline 1.01 & Movilización y Desmovilización & $15 / 09 / 2016$ & $24 / 10 / 2016$ & Semana 03 & Semana 09 & Sí \\
\hline 2.00 & \multicolumn{6}{|c|}{ Conservación de Plataforma y taludes } \\
\hline 2.01 & $\begin{array}{c}\text { Limpieza de Derrumbes y Huaycos } \\
\text { Mayores }\end{array}$ & $29 / 09 / 2016$ & $29 / 09 / 2016$ & Semana 05 & Semana 05 & Sí \\
\hline 3.0 & \multicolumn{6}{|c|}{ Conservación de Calzada en afirmado } \\
\hline 3.01 & Bacheo de Afirmado & $28 / 09 / 2016$ & $18 / 10 / 2016$ & Semana 05 & Semana 08 & Sí \\
\hline 3.02 & $\begin{array}{l}\text { Perfilado de la Superficie sin Aporte } \\
\text { de mat. }\end{array}$ & $16 / 09 / 2016$ & $20 / 10 / 2016$ & Semana 03 & Semana 08 & Sí \\
\hline 4.00 & \multicolumn{6}{|c|}{ Conservación de Drenaje superficial } \\
\hline 4.01 & Limpieza de Cunetas & $26 / 09 / 2016$ & $20 / 10 / 2016$ & Semana 05 & Semana 08 & Sí \\
\hline
\end{tabular}




\section{- Nivel 3. Planificación semanal}

Lo que se "Hará" son las actividades sin restricciones seleccionadas del ITE que se incluyen en la planificación semanal (semana 00 - semana 10). Contempla los siguientes criterios:

- Confección del programa semanal

Con la programación semanal diaria (semana 00 - semana 10); especificando el metrado de avance y las progresivas de ubicación del trabajo a lo largo del tramo.

- Compromisos a ejecutar actividades

Se establecen las responsabilidades que corresponden al Asistente y al Capataz; para que la planificación semanal cumpla con los objetivos.

\section{Grupo de procesos de monitoreo y control}

Gestión del cronograma del proyecto (Planificación

\section{Last Planner)}

c1. Controlar el cronograma (Planificación Last Planner)

- Control de los planes de trabajo semanal

Gestión de los costos del proyecto

d1. Controlar los costos (Last - Planner - Control y mejoramiento continuo)

- $\quad$ Porcentaje de programación cumplida (PPC)

Para estimar la efectividad, se realizó la medición del desempeño de cada plan de trabajo semanal; mediante la siguiente fórmula.

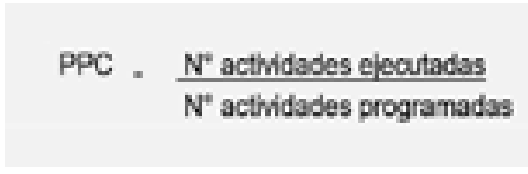

\section{RESULTADOS}

En la Tabla 5 y en la Figura 3, se consignan e ilustran; los PPC de la semana 00 a la semana 10 .

Tabla 5: Porcentaje de programación cumplida (PPC) por semana. Fuente: Elaboración propia.

\begin{tabular}{ccccc}
\hline Semana & $\begin{array}{c}\mathrm{N}^{\circ} \text { actividades } \\
\text { programadas } \\
(\mathrm{a})\end{array}$ & $\begin{array}{c}\mathrm{N}^{\circ} \text { actividades } \\
\text { ejecutadas } \\
(\mathrm{b})\end{array}$ & $\mathrm{PPC}=\mathrm{b} / \mathrm{a}$ & $\begin{array}{c}\mathrm{PPC} \\
\%\end{array}$ \\
\hline Semana 00 & 9 & 9 & 1 & 100 \\
Semana 01 & 8 & 8 & 1 & 100 \\
Semana 02 & 3 & 3 & 1 & 100 \\
Semana 03 & 6 & 6 & 1 & 100 \\
Semana 04 & 5 & 5 & 1 & 100 \\
Semana 05 & 7 & 7 & 1 & 100 \\
Semana 06 & 4 & 4 & 1 & 100 \\
Semana 07 & 4 & 4 & 1 & 100 \\
Semana 08 & 7 & 7 & 1 & 100 \\
Semana 09 & 5 & 4 & 0.8 & 80 \\
Semana 10 & 5 & 5 & 1 & 100 \\
\hline
\end{tabular}

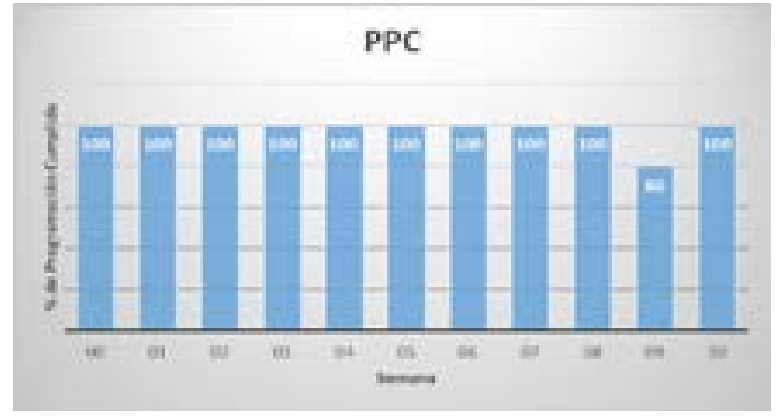

Fig. 4: Porcentaje de Plan Cumplido. Fuente: Elaboración propia

En la semana 09, se tenía programada la Desmovilización de equipo mecánico, para el 24/10/2016, la que no se ejecutó, excepto las otras 4 otras actividades programadas; por lo que corresponde un PPC del $80 \%$. La actividad fue reprogramada para la semana $10(31 / 10 / 2016)$. Se dio una Causa de No Cumplimiento, por la no disponibilidad de cama baja (Desmovilización de equipo mecánico); habiéndose reprogramado la actividad.

\section{DISCUSIÓN Y CONCLUSIONES}

a. La aplicación del Lean Construction, permitió realizar un análisis de los tiempos productivos, tiempos contributorios y no contributorios; con el objetivo de determinar la eficiencia del proceso de Perfilado de la subrasante sin aporte de material e implementar las mejoras necesarias. (Figura 3).

b. Al incluir el escenario Last Planner al Grupo de procesos de planificación del PMBOK, se logró realizar una planificación más real con actividades sin restricciones que fueron consideradas en la programación semanal diaria; generando mayor confiabilidad.

c. Asimismo, al incluir el Last Planner en el Grupo de procesos de monitoreo y control, se logró estimar la efectividad mediante la medición del desempeño de cada plan de trabajo semanal, a través del porcentaje de procesos completados (PPC); que permitió identificar las causas de no cumplimiento (CNC) e implementar las mejoras que se reflejan en el logro de la ejecución del $100 \%$ de las actividades programadas.

d. La optimización del proceso de Perfilado de la subrasante sin aporte de material generó grandes resultados (outputs) como la reducción del tiempo (plazo de ejecución) de 98 días a 68 días y la reducción del costo de S/. 255,851.59 a S/. 230,061.70; estos outputs son por unidad de consumo (inputs) que corresponde a los recursos administrados eficientemente (Mano de obra, equipo y materiales) y a un eficaz control de calidad. (Tabla 2).

e. En base a lo indicado en los literales c) y d), se puede concluir que la conjunción de la eficacia y la eficiencia en la aplicación de la metodología propuesta, mejoro significativamente la productividad del mantenimiento rutinario del proyecto. 


\section{REFERENCIAS BIBLIOGRÁFICAS}

1. La aplicación del Lean Construction, permitió realizar un análisis de los tiempos productivos, tiempos contributorios y no contributorios; con el objetivo de determinar la eficiencia del proceso de Perfilado de la subrasante sin aporte de material e implementar las mejoras necesarias. (Figura 3).

2. Al incluir el escenario Last Planner al Grupo de procesos de planificación del PMBOK, se logró realizar una planificación más real con actividades sin restricciones que fueron consideradas en la programación semanal diaria; generando mayor confiabilidad.

3. Asimismo, al incluir el Last Planner en el Grupo de procesos de monitoreo y control, se logró estimar la efectividad mediante la medición del desempeño de cada plan de trabajo semanal, a través del porcentaje de procesos completados (PPC); que permitió identificar las causas de no cumplimiento (CNC) e implementar las mejoras que se reflejan en el logro de la ejecución del $100 \%$ de las actividades programadas.

4. La optimización del proceso de Perfilado de la subrasante sin aporte de material generó grandes resultados (outputs) como la reducción del tiempo (plazo de ejecución) de 98 días a 68 días y la reducción del costo de S/. 255,851.59 a S/. 230,061.70; estos outputs son por unidad de consumo (inputs) que corresponde a los recursos administrados eficientemente (Mano de obra, equipo y materiales) y a un eficaz control de calidad. (Tabla 2).

5. En base a lo indicado en los literales c) y d), se puede concluir que la conjunción de la eficacia y la eficiencia en la aplicación de la metodología propuesta, mejoro significativamente la productividad del mantenimiento rutinario del proyecto. 